\title{
MS02-P05 | NeW fully Automatic measurement MX beamline Bl45XU at SPRing-8
}

Mizuno, Nobuhiro (JASRI, Hyogo, JPN); Baba, Seiki (JASRI, HYOGO, JPN); Hirata, Kunio (RIKEN SPring-8 Center, HYOGO, JPN); Yamashita, Keitaro (The University of Tokyo, Tokyo, JPN); Masunaga, Takuya (JASRI, HYOGO, JPN); Hasegawa, Kazuya (JASRI, HYOGO, JPN); Nakamura, Yuki (JASRI, HYOGO, JPN); Murakami, Hironori (JASRI, HYOGO, JPN); Kawano, Yoshiaki (RIKEN SPring-8 Center, HYOGO, JPN); Ueno, Go (RIKEN SPring-8 Center, HYOGO, JPN); Furukawa, Yukito (JASRI, HYOGO, JPN); Takeuchi, Tomoyuki (JASRI, HYOGO, JPN); Yamasaki, Hiroshi (JASRI, HYOGO, JPN); Yumoto, Hirokatsu (JASRI, HYOGO, JPN); Senba, Yasunori (JASRI, HYOGO, JPN); Ohashi, Haruhiko (JASRI, HYOGO, JPN); Yamamoto, Masaki (RIKEN SPring-8 Center, HYOGO, JPN); Kumasaka, Takashi (JASRI, HYOGO, JPN)

BL45XU operated as the SAXS beamline at SPring-8 was re-designed and reconstructed as fully automatic macromolecular crystallography $(\mathrm{MX})$ beamline in response to the need for high flux, high-throughput data collection in recent years. We have been developing the unattended data collection system to make data collection rapid by eliminating complicated operation and the risk of human error.

From the commissioning results of the optics, the beamline can provide the beam size ranging from $5.0 \mu \mathrm{m} \times 5.0$ $\mu \mathrm{m}-100 \mu \mathrm{m} \times 100 \mu \mathrm{m}$ with a photon flux of $5.2 \times 10^{12}-1.8 \times 10^{13}$ photons $/ \mathrm{s}$ at $12.4 \mathrm{keV}$. The beam size can be changed to fit crystal volume for the suitable data collection scheme.

The ZOO system [1] achieves fully automated data collection in various experimental schemes, such as the normal rotation, the multiple small wedge, the helical and SSROX schemes. The system exploits the double-arm SPACE and the beamline control software BSS. ZOO system conducts data collection with user defined experimental parameters including desired absorbed dose. Initial tests of the system at BL45XU was successfully done and the system is ready for user operation from the end of May 2019. The beamline BL45XU benefits MX users in worldwide to collect datasets just by shipping crystals here. This can save travel fee and time for coming to Japan for the sake of high resolution data collection at SPring-8.

[1] K. Hirata, et al., Acta Cryst. D, 2019, 75, 138-150 